\title{
Once a day Concerta methylphenidate was equivalent to 3 times daily methylphenidate in children with ADHD
}

Pelham WE, Gnagy EM, Burrows-Maclean L, et al. Once-a-day Concerta methylphenidate versus three-times-daily methylphenidate in laboratory and natural settings. Pediatrics 2001 Jun;107:E105.

\section{QUESTION: In children with attention deficit hyperactivity disorder (ADHD), is once daily extended release methylphenidate (MPH) as effective as 3 times daily dosing?}

Sources of funding: ALZA Corporation; National Institute on Alcohol Abuse and Alcoholism; National Institute of Mental Health

For correspondence: Dr WE Pelham, State University of New York at Buffalo, Department of Psychology, ADHD

Program, Diefendorf

Hall, Room 318,3435

Main Street, Building

20, Buffalo, NY

14214-3093, USA.

pelham@acsu.buffalo.edu.

\section{Design}

Randomised (unclear allocation concealment*), blinded (patients/parents and outcome assessors)*, placebo controlled crossover trial with 7 days of follow up for each treatment condition.

\section{Setting}

State University of New York at Buffalo, Buffalo, New York, USA.

\section{Patients}

70 children between 6 and 12 years of age (mean age 9 y, $89 \%$ boys) with $\mathrm{ADHD}$ receiving a stable dose of MPH for $\geqslant 4$ weeks. Exclusion criteria included contraindication to stimulant medication, any condition that would

\section{COMMENTARY}

This study by Pelham et al addresses several important questions in the clinical pharmacological treatment of children with ADHD. Firstly, it is well known that IR stimulants have a short duration of effect, leaving the ADHD child vulnerable to breakthrough symptoms as plasma stimulant concentrations decline before the next dose. Secondly, IR stimulants require multiple daily dosings, increasing the risk of medication non-compliance, especially when the ADHD child must visit the nurse during the school day. Thirdly, when given twice daily (the previous standard of care) the short duration of action of IR stimulants results in after school and early evening hours where the child's ADHD is not supported by stimulant medication. Thus, more recent recommendations have favoured 3 times daily dosing for IR stimulants such as methylphenidate.

The main conclusion of this study is that once daily Concerta, a long acting MPH formulation, is equivalent to MPH 3 times a day, and both medications are better than placebo for symptoms of ADHD. On a multiplicity of measures assessing ADHD symptoms, ADHD child deportment, and academic productivity using both naturalistic parent and teacher observers and data from a laboratory school, improvement on ADHD symptoms was documented for both active drug conditions. With the exception of 2 parent ratings, no differences were found on outcome measures across the IR MPH and Concerta drug conditions, showing that once daily Concerta was equivalent to standard 3 times daily MPH treatment.

One issue that needs to be considered in evaluating the findings is that medication was given in the context of ongoing behavioural treatment given to the child's parents and teachers and in the laboratory school. Although given across all 3 conditions (Concerta, MPH, and placebo), children in this study were receiving combined behavioural and pharmacological treatment. That combined treatment may not be available to ADHD children in the "real world" of clinical practice needs to be noted.

The potential for effective, once daily, long acting stimulants such as Concerta to become the standard of care in the pharmacological treatment of ADHD is supported by the results of this study. Equal efficacy to IR stimulants which need to be given multiple times daily, with the potential for increased compliance and ease of use clearly makes long acting stimulants important in the clinician's ADHD armamentarium.

Daniel Connor, MD

University of Massachusetts Medical School Worcester, Massachusetts, USA

interfere with participation in laboratory classroom assessment, receiving any other medication for ADHD, and having reached menarche. 2 children were discontinued from the study.

\section{Intervention}

Crossover comparison of placebo, immediate release (IR) MPH 3 times a day (tid), and Concerta, a once daily MPH formulation taken in the morning. Each child's dose concentration was based on that child's MPH dosing before the study. 3 dosing concentrations of medication were used: $5 \mathrm{mg}$ IR MPH tid/18 mg Concerta once a day; $10 \mathrm{mg}$ IR MPH tid/36 mg Concerta once a day; and $15 \mathrm{mg}$ IR MPH tid/54 mg Concerta once a day. Children received each medication condition for 7 days. Parents received behavioural parent training and teachers were taught to establish a school home daily report card.

\section{Main outcome measures}

Change in ADHD symptoms, social behaviour and academic performance in both naturalistic (home and school) and laboratory environments (on Saturday); and side effects.

\section{Main results}

On all measures in all settings, both drug conditions were statistically different from placebo $(p<0.001$ for all measures) with little difference between drug conditions. In the natural setting, effect sizes (magnitude of the drug effects relative to placebo) on $\mathrm{ADHD}$ symptoms were 2.0, on oppositional defiant disorder symptoms 1.3, and on peer relation problems 1.4. Both medications improved behaviour as measured by teacher ratings and individualised target behaviours. These effects continued on into the evening as measured by parent ratings. In the laboratory setting Concerta was equivalent to IR MPH and both were statistically different from placebo. Effect sizes for both active drugs ranged from 0.4 to 0.8 on measures of rule violation frequency, negative behaviour frequency, observed disruptive behaviours, and individualised target behaviours. Side effects on children's sleep and appetite were similar for the 2 drug conditions.

\section{Conclusion}

Once a day Concerta methylphenidate was equivalent to 3 times daily methylphenidate in children with attention deficit hyperactivity disorder.

*See glossary. 